\title{
Limitations of glycated haemoglobin (HbA1c) in Sulaimani diagnostic laboratories
}

\author{
Dereen Najat \\ Chemistry Dept, College of Science \\ Sulaimani University \\ Sulaimani, Iraq \\ Dereen.mohammad@univsul.edu.iq
}

\begin{abstract}
HbAlc is the test of choice in many countries to monitor and diagnose diabetes. There are continuing debates on the HBAlc usage. HBAlc has some limitations, for example, HBAlc test should not be used in pregnant women and children under the age of 13, vitamin B12 deficiency and Hypertriglycemia increases HBAlc levels, many other conditions such as iron deficiency anemia, stage 4 or 5 chronic kidney disease, hemoglobinopathy all interfere with HBAlc results. Moreover, sensitivity and specificity of the test vary among different ethnic groups. In this study, we aimed to investigate the knowledge of health professionals on HBAlc test limitations and to assess the frequency of HBAlc usage in the pediatric population. We also assessed the quality of the instruments used in Sulaimani laboratories (labs) to evaluate HBA1c. One hundred (100) diagnostic labs in Sulaimani city was surveyed to determine HBAlc instruments types used in labs. A one-page questionnaire on the practice and HBAlc limitations test was given to 100 health professionals. Most of the health professionals in this study were unaware of the limitations of the HBAlc test, $99 \%$ of the physicians were unaware of the inaccuracy of the point of cares testing (POCT) devices and the frequency of POCT uses in Sulaimani diagnostic labs. Surprisingly, Sulaimani Pediatric hospital used the HBAlc test to monitor and diagnose type 1 diabetes patients. Our survey also showed Cobas C111 analyzer was the most common (47.8\%) type of instrument used in Sulaimani city. the advantage was used by $8 \%$ of the labs, Biohermes and auto accent was used by $4.3 \%$ of labs. Surprisingly, the Diabetes and Endocrine Center in Sulaimani used the POCT device SD AlcCare instrument. In conclusion, almost all health professionals were unaware of the HBAlc limitations. Continuing education programs to all health professional should be mandated in Sulaimani city hospitals to ensure high quality of the HBAlc test. Most importantly, health professionals need to be aware that POCT is widely used in Sulaimani labs and that POCT is considered an inaccurate method for HBAlc evaluation. Pediatric physicians need to be reminded that HBA1c is not recommended in children.
\end{abstract}

Keywords: point of care testing, $\mathrm{HbAlc}$, diabetic mellitus, quality control, limitations.

\section{INTRODUCTION}

Glycated hemoglobin is the test of choice for monitoring glycemic index in diabetic patients. Low HbA1c levels are associated with lower microvascular complications in type 1 and type 2 diabetes mellitus [1].
HbA1c basically reflects the amount of sugar bound to hemoglobin for the past three months [2]. Practically, it has many advantages over other tests because it is more stable than other analytes, it is not affected by fasting state or other pre-analytical errors. Despite the many advantages of HbA1c test, but it has serious limitations which are often overlooked by health professionals. $\mathrm{HbA} 1 \mathrm{c}$ has both clinical and experimental limitations and there are many situations where using $\mathrm{HbAlc}$ alone for either diagnosing or monitoring glycemic control is not preferred and might cause serious complications [3]. Small changes in HbA1c levels, 1\%, can have huge impact on patients overall health, this $1 \%$ HbA1c change might cause $14 \%$ decrease in myocardial infarction, 37\% decrease in microvascular complications and $21 \%$ down in death by diabetes. Therefore, it is very crucial to be careful when measuring $\mathrm{HBa} 1 \mathrm{c}$ levels and it is important to use accurate methods of determination [4].

One of the issues of $\mathrm{HbAlc}$ is that there are ethnic differences in hemoglobin types and there are about 1175 types of hemoglobin variants, about $7 \%$ of global populations have different hemoglobin variants, this variation causes interferences with $\mathrm{HbA1c}$ results [2].

Conditions such as hemolytic anemias, blood loss or multiple transfusions or any other conditions which shorten lifespan of red blood cells (RBC) will falsely lower HbA1c levels. However, not all anemias will cause low HbA1c levels, conditions causing longer RBC lifespan will cause falsely higher $\mathrm{HbA1c}$ levels, these conditions include pernicious anemia and iron deficiency anemia [2].

Furthermore, splenomegaly, rheumatoid arthritis or any other drugs or diseases which might cause short RBC lifespan all causes low HbA1C levels. Chronic disease also shorten RBC lifespan and causes false low HbA1c levels however when jaundice produces raised bilirubin levels this also cause false levels of Hba1c levels [2]. In addition to the above mentioned conditions causing false HbA1c results, there are many other mechanism which might cause interferences with HbA1c analysis. For example, factors affecting the glycation of glucose to hemoglobin, vitamin $\mathrm{C}$ and vitamin $\mathrm{E}$ inhibit glycation and hence lower HbA1c levels. Although the mechanism is still unknown, it has been shown that chronic alcoholism also causes lower false HbA1c levels [2]. These false results might cause false treatment for diabetic patients and might cause serious complications. In addition to interferences caused by biological variables, instruments used to measure $\mathrm{HbA1c}$ also produce false HbA1c levels. Point of care testing (POCT) devices, although inaccurate, are used 
commonly specially in developing countries mainly because of the lower costs of POCTs [5]. Until now there is no global agreement to harmonize HbA1c testing. Different methods give variable accuracies, with the POCT devices being the least accurate, followed by immunoassays (coefficient of variation, C.V=5-6\%), high performance liquid chromatography (C.V=2-3\%) and mass spectroscopy being the most accurate method. In 2009 Erna Lenters-Westra etal investigated the performance of common HbA1c POCTs devices and reported that 6 out of 8 POCTs devices didn't meet the accepted performance criteria of $<3 \% \mathrm{CV}$, since that study, many company started to withdraw their devices or release modified instruments, this action shows that POCTs are generally unreliable and rigorous quality control measure should be used on POCT. Another study by the same group in 2014 also showed that three out of 7 devices are still not reliable, these manufactures included Quo-test, Quo-lab, innovastar and many others. A study in Germany concluded that among four different labs, there were considerable variations in the $\mathrm{HbA} 1 \mathrm{c}$ levels performed on 75 patients [6]

Moreover, it is still under debate to whether it is relevant to use $\mathrm{HbA} 1 \mathrm{c}$ in diagnosis, especially in pregnant and pediatric population, a recent study showed that negative bias was evident in these populations and $\mathrm{HbA} 1 \mathrm{c}$ is more likely should be used to management of diabetic and monitor glycemic index rather than a tool for diagnosing diabetes [7].

Based on these controversies around the HbA1c usage in both diagnosis and the biological and instrumental limitations of $\mathrm{HbA} 1 \mathrm{c}$ devices, we sought to estimate the prevalence of POCTs devices in selected Sulaimani city diagnostic labs; we also investigated the knowledge, awareness of health professionals especially physicians on the limitations of HbA1c and the frequency of POCT usage among physicians in Sulaimani city.

\section{MATERIALS AND METHODS}

\section{Study design}

100 diagnostic labs in Sulaimani city were surveyed to determine HBA1c instruments types. We surveyed both public and private diagnostic labs. The type of instrument used by each lab was recorded and photographed.

The percentage of usage was expressed as \% for each instrument used. A diverse group of Health professionals (nurses, laboratory technicians, phlebotomists, lab scientists and physicians-including registrars, consultants, those without postgraduate degree and those with boards, PhDs.. etc) were give a questionnaire. Each of the precipitants gave consent.

The questionnaire asked respondents about their occupation, age, place of work and duration of work, year of graduation and in case of doctors, whether they finished their residency program. Further, the health professionals were asked about their knowledge and attitude toward POCT devices. Physicians were asked if they had personal POCT at their offices, their knowledge about the biological and instrumental limitations of HBA1c test, limitations of POCT devices; whether they are aware of the type of the instruments used in lab and how important is sensitivity and selectivity of the instruments. Paediatric doctors were asked about their practice limitations of HbAlc test in paediatric population.

\section{HbA1c determination}

108 Diabetic Children aged 6-13 years old were included in this study.HbA1C results were obtained from pediatric diabetic center. Blood samples were withdrawn from diabetic patients. A verbal consent were obtained from the parents of the children. Blood samples were transferred in to Ethylenediaminetetraacetic acid (EDTA) vaccutainers and then whole blood samples were analysed with Cobas C111 analyser (Immunoassay method using Cobas c 111- Roche Diagnostics GmbH, Germany).

\section{RESULTS}

80 diagnostic labs (73\% private labs and $27 \%$ public labs) in Sulaimani city were surveyed to determine HbA1c instruments types. 170 health professionals were asked to participate in HbA1c limitations awareness survey. Lost and incomplete data were not considered in the final results, the final completed data was from 100 health professionals and 50 diagnostic labs.

Point of care devices are largely used, SD A1ccare instrument, which is used at Sulaimani diabetic and endocrine center, was used by $1 \%$ of labs. (7\%) of private labs did not perform the test at the time of data collection. Autoaccent, Biohermes, Biolabo were used by $1 \%$ of diagnostic labs.

Cobas C111, was most commonly used instrument (11\%), Figure 1.

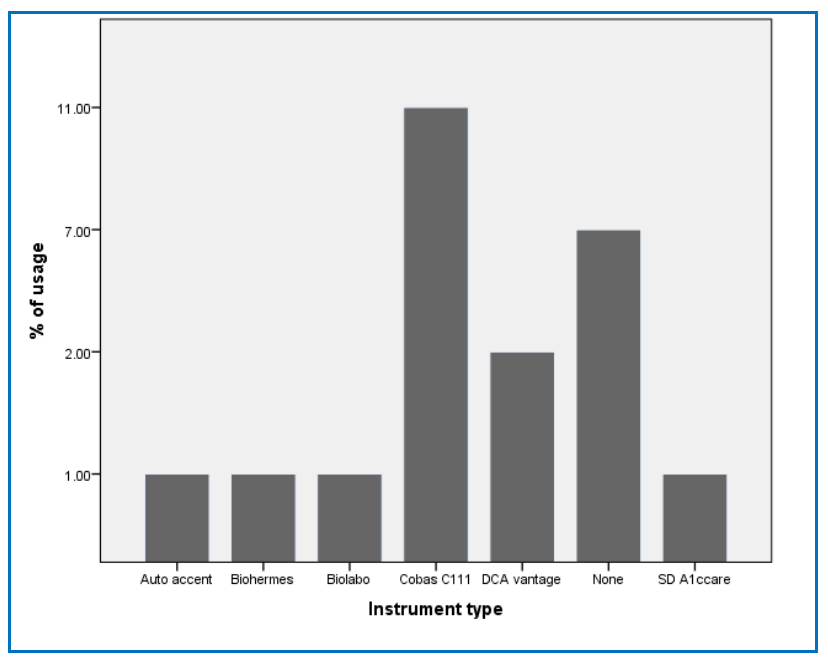

Fig1.Types and percentage of instruments used in Sulaimani diagnostic labs 
The questionnaire looked in to state of point-of-care devices at Sulaimani health centers, the questionnaire were given to doctors with various specialties at various health centers in Sulaimani city. $3 \%$ of doctors owned personal POCT analyzers. Only $57 \%$ of the doctors were knowledgeable about the limitations of POCT devices and only $1 \%$ were aware of the common types of $\mathrm{HbA1c}$ instruments used in the labs. none of pediatrician were aware of the limitations of HbAlc usage in pediatric population.

Table1. Awareness, Knowledge, and Experience of Point-of-Care Devices of Doctors

\begin{tabular}{ll}
\hline \hline Level of awareness & \% \\
\hline$\%$ of doctors with POCT analyzers & 3
\end{tabular}

\begin{tabular}{ll}
\hline$\%$ of doctors knowing limitations of HbA1c & 57 \\
\hline$\%$ of doctors knowing the limitations of POCT & 8 \\
\hline$\%$ of doctors knowing types of HbA1c devices used & 1 \\
\hline $\begin{array}{l}\% \text { of doctors knowing misuse of HbA1c in paediatric } \\
\text { population }\end{array}$ & 0 \\
\hline
\end{tabular}

Finally, we investigated the use of HbA1c levels in pediatrics population and found out that the majority of diabetic children (6-13 years old, $n=108)$ are monitored through the HbAlc tests, surprisingly the average HbA1c levels were 10.85 in diabetic children aged between 6-13 years old.

\section{DISCUSSION}

In the past years, there has been continuous debate on HbA1c usage and its limitations.

HbA1c limitations are mainly caused by many biological factors, which causes false results also, limitations of the instruments used to measure $\mathrm{HbAlc}$ levels cause misleading results [2]. In this study, we looked in to the type of the instruments used in diagnostic labs in Sulaimani city. Most physicians report inaccurate results of HbA1c testing and they complaint that they have to repeat $\mathrm{HbAlc}$ tests at least at three different labs to confirm the final results. The main problem with the HbA1c results is that is even with small changes in HbaA1c levels, physicians has to change the course of treatment, as a result the limitations of $\mathrm{HbAlc}$ are both life threating and costly [8]. In the past few years and because of the financial problems hospitals and diagnostic labs reduced their budget, and since POCT are less expensive than the other detection methods, POCT has spread widely in the city, therefore, we surveyed the types and frequency of instruments used in Sulaimani diagnostic labs. Our results showed that $7 \%$ of labs didn't perform the test in the first place because of the high costs of the test. Whereas Cobas C111, which is regarded as an accurate method was used by $11 \%$ of the labs. POCTs such as Biohermes, Autoaccent and Biolabo were used equally by $1 \%$ the labs. DCA vantage and SD Care was used by $1 \%$ of labs, Whereas the DCA vantage is regarded as an accurate POCT, SD care is regarded one of the best POCT devices in the market. However, none of the POCTs should be used on a continuous bases and final results should always be confirmed at least by immunoassays or by HPLC methods [9].

The second part of our study looked in to the awareness and knowledge of physicians and health professionals on the types of the instruments used in diagnostic labs and whether they had awareness on the limitation of $\mathrm{HbA} 1 \mathrm{c}$ test, surprisingly a high percentage of the doctors were unaware of both of biological and the instrumental limitations. Only $1 \%$ of the physicians had knowledge about the types and accuracies of the instruments used and they were unaware about the limitations of POCT devices. Our investigation also revealed that most pediatric physicians were unaware on the limitations of $\mathrm{HbA1c}$ and that public pediatric hospitals use HbA1c frequently. This result should prompt health authorities to raise awareness on the limitations of $\mathrm{HbA} 1 \mathrm{c}$ in pediatric population [7]. Surprisingly, the results for HbA1c in 108 diabetic children aged 6-12 showed an average as high as 10.58 , only three children had normal HbA1c levels. This high HbA1c value is alarming, and most likely resulted from pre-analytical or analytical lab errors [10], our results highlights the importance of accurate devices usage and the urgent need to put POCT devices used in Sulaimani diagnostic hospitals to strict quality control measures and strict monitoring from international quality control programs.

Our results are limited to a small number of health professionals and younger physicians who are not specialists in internal medicine, this might explain the high percentage of ignorance on HbAlc limitations, we didn't gather information from consultants in internal medicine because they were mostly uncooperative to respond to our questionnaire, therefore, we asked non specialist doctors in our survey. The study was performed on small number of labs and to get conclusive results, we need to expand the number of the labs we surveyed and also reach out for other Kurdish cities in the region.

Another limitation of the study is that the time of the survey was in 2017 , which is marked by high economic instability in the region, which prompted the popularity of POCT devices.

Based on our results, we recommend proficiency testing should be mandated for HbA1c POCT users to ensure quality. The ministry of health and in collaboration with universities need to raise awareness among health professionals in general and physicians in particular on the limitations of HbA1c test and the frequency of POCT devices in diagnostic labs. Health professional should undergo continuous educational programs to ensure that 
physicians and health professional are updated with the latest information and advancement in the field [11].

\section{CONCLUSION}

Most of health professionals were unaware of the biological and instrumental limitations of HbA1c test; POCT devices are widely used in Sulaimani labs.Sulaimani Pediatric hospital uses Hb1AC test to monitor diabetes in children.Most importantly, health professionals need to be aware that and that POCT is considered an inaccurate method for HbAlc determination. These results indicates that not only health professionals but also policy makers should always consider the limitations of $\mathrm{HbAlc}$ test before making judgment on glycemic control, otherwise overtreatment or mistreatment of diabetic patients based on false HbA1c levels will have serious health complications

\section{ACKNOWLEDGEMENT}

We are grateful for Paediatric diabetic centre at Sulaimani Paediatric hospital for their huge support during our research, we can't thank them enough. We also would like to thank all the participating hospitals and health professionals. Special thanks to chemistry students for their efforts, without them this research was not possible

\section{REFERENCES}

[1] MJL. Hare, JE. Shaw, PZ. Zimmet, "Current controversies in the use of haemoglobin A1c," J Intern Med, vol. 271, pp. 227-36, 2012.

[2] SI. Sherwani, HA. Khan, A. Ekhzaimy, A. Masood, MK. Sakharkar, "Significance of HbA1c Test in Diagnosis and Prognosis of Diabetic Patients," Biomark Insights, vol. 11, pp. 95-104, 2016.

[3] R. Hellman, "When are HBA1C Values Misleading?," AACE Clin Case Reports, vol. 2, pp. 377-9, 2016.

[4] IM. Stratton, AI. Adler, HAW. Neil, DR. Matthews, SE. Manley, CA. Cull, et al., "Association of glycaemia with macrovascular and microvascular complications of type 2 diabetes (UKPDS 35): prospective observational study," BMJ vol. 321, pp. 72, 2000.

[5] E. Lenters-Westra, RJ. Slingerland, "Three of 7 Hemoglobin A1c Point-of-Care Instruments Do Not Meet Generally Accepted Analytical Performance Criteria," Clin Chem, vol. 60, pp. 1062-72, 2014.

[6] J. Roth, N. Müller, T. Lehmann, K. Böer, S. Löbel, J. Pum, et al, "Comparison of HbA1c Measurements using 3 Methods in 75 Patients Referred to One Outpatient Department," Exp Clin Endocrinol Diabetes, 2017.

[7] JL. Clark, L. Rao, "Retrospective Analysis of Point-of-Care and Laboratory-Based Hemoglobin A1c Testing," J Appl Lab vol.1, 2017.
[8] L. Heinemann, G. Freckmann, "Quality of HbA1c Measurement in the Practice: The German Perspective," J Diabetes Sci Technol, vol. 9, pp. 687-95, 2015.

[9] J. Szymezak, N. Leroy, E. Lavalard, P. Gillery, "Evaluation of the DCA Vantage analyzer for HbAlc assay," Clin Chem Lab Med, vol. 46, pp. 1195-8, 2008.

[10] O. Sonntag, "Analytical interferences and analytical quality," Clin Chim Acta, Jun, vol. 404, pp. 37-40, 2009.

[11] M Fletcher, "Continuing education for healthcare professionals: time to prove its worth," Prim Care Respir J, vol. 16(3), pp. 188-90, 2007. 